\title{
States and properties of metallic systems at a threshold breakdown of the through holes under power laser action (part 2). Susceptibility
}

\author{
Kalashnikov $E^{1 a}$., Kantor $M^{2}$., Bugayev $A^{3}$. \\ ${ }^{1}$ Moscow Region State University, Moscow, Russia \\ ${ }^{2}$ Ioffe Physical Technical Institute, RAS, S.-Petersburg, Russia \\ ${ }^{3}$ Applied Research Center Old Dominion University, VA 23606 USA
}

\begin{abstract}
Threshold breakdown of the through holes by power laser radiation of metallic foils is considered as response of metallic system to laser radiation. Binding experimentally determined response to the absolute temperature scale allows to determine the value of the imaginary part of the generalized susceptibility depending on temperature, the critical temperature of the transition "liquid metal - gas", states of the electronic subsystems at this temperature, and the reflectance coefficient values. Key words: Response, imaginary part of the generalized susceptibility, plasm frequency, frequency of electron-ion interaction, critical temperature of second order "liquid metal-gas" phase transition, reflectance coefficient.
\end{abstract}

\section{Introduction}

One of measuring in an experiment on the threshold holes of metallic foils under act of powerful laser radiation is a diameter of threshold exit hole [1]. - The diameter is absent if the influence of external fields of laser radiation is absent or is not enough. In other words, minimum diameter of threshold exit hole is, not that another, as response of the system to an external action [2]. A size of diameter of exit hole, in the created conditions, is fluctuating value under act of the external field. Our case, just gives possibility to connect properties of fluctuating magnitude with values characterizing behavior of body under external action [2]. Formally, response $d$ is expressed through susceptibility operator $\alpha[2]$ :

$$
d=Q f
$$

here $f$ - generalized force is time function

\section{Model}

The falling on a target the electromagnetic field of laser radiation interacts with the electronic subsystem of metal and enlarges in region of this interaction a chemical potential $\mu_{e}$ of electrons. (Pondermotive potential [3] $V=\left(q^{2} / 4 c \cdot m \cdot \omega^{2}\right) \cdot E^{2}$ is about $10^{-6} \div 10^{-4} \mathrm{eV}$ at created conditions $\lambda=694 \mathrm{~nm}$ and intensity $J \sim 10^{6} \div 10^{10} \mathrm{~W} / \mathrm{cm}^{2}$ ) Therefore electrons will begin to move into place, where their chemical potential is smaller or there (what is the same) where their number (density) is less. Into a metal, their density remains unchanged. Therefore, electrons begin the motion into place, where them less - from a metal to the interface of "metal-gas". Such motion, in particular, arises up, when a solid is primary quickly heated to the very high temperatures [4]. Kinetic energy of motion of electrons and ions at these densities (densities of metals in solid and liquid states) turns out comparable with coulomb energy of their interaction. Electrons are concentrating at a "gas-metal" interface. It leads to original polarization at a "interface" which is expressed as difference of chemical potentials:

$$
\left(\mu_{e_{-} \text {interface }}-\mu_{e_{-} \text {volume }}\right)=e \cdot\left(\varphi_{\text {interface }}-\varphi_{\text {volume }}\right)
$$

here $\mu_{e_{-} \text {interface }}$ and $\mu_{e_{-} \text {volume }}$ is chemical potentials of electrons on interface and in volume. Therefore, the arising up here electric field of $E$

$\left(\partial \varphi / \partial r=(1 / e) \cdot \partial \mu_{e} / \partial r=-E ; \quad \mu_{e}=\varepsilon_{F e r m i}\right.$, for cooper $\varepsilon_{\text {Fermi }}=7 \mathrm{eV}$ so that the order of magnitude of electricfield tension corresponds to $-E \sim 7.10^{8} \mathrm{~V} / \mathrm{cm}^{2}$ ) drags out positive ions from a warmed-up volume. Accelerated ions, passing through the "gas-metal" interface and, due to comparableness of kinetic energy of motion and coulomb energy of interaction, capture electrons, and abandon a metal. 


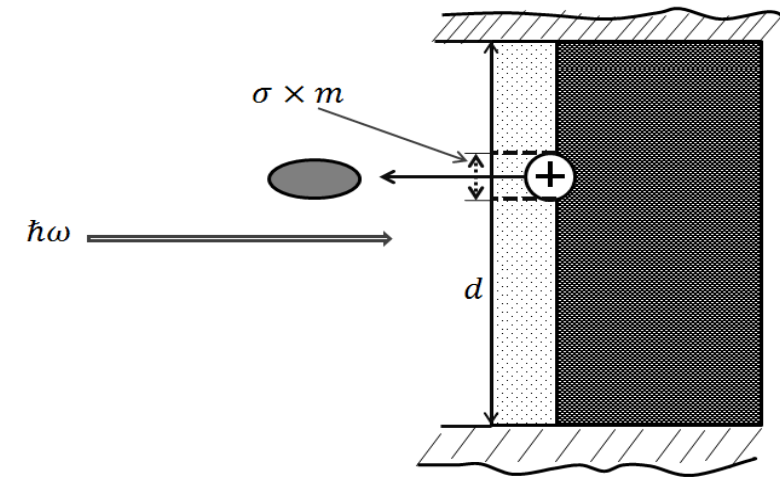

Fig. 1. Creation (annihilation) of element of interface, $\sigma$, on leaving of ion from the volume of metal

Therefore, an ion, passing through an interface, participates in formation of surface, increasing (or reduction) its area, fig.1 Therefore, an ion, passing through an interface and transforming itself into atom, is the same fluctuation part of surface which is the response of the system on the external field $f$, (appendix of A).. Therefore, the elemental act of displacement (crossing of interface) can be considered as an act of moving away (joining) of element of interface area per time. This element of area, $\sigma$, is approximately equal to the "area of surface» of shade of atom (appendix of A). Elemental area, $\sigma$, per time is connected with mass, $m$, of atom. It is therefore possible to talk about moving away (formation) per time of area in the interface, by the characterized composition $N \cdot \sigma \cdot m$, where $N$ is an amount of atoms in this region. Then value $z$ measureable in an experiment on the threshold breakdown of the through holes is connected to the mean value of $d$ (appendix of A)

$$
N \cdot \sigma \cdot m=\pi \cdot(d / 2)^{2} \cdot m=(\pi / 4) \cdot(z \cdot \sqrt{ } m)^{2}
$$

Effective diameter of the threshold exit hole, $d=z \cdot \sqrt{ } m$, is the response of the system on external action $f$. As a threshold diameter of $z$ develops in time, then, in general case, $d$ is the function of time of $t$ :

$$
d=d(t)
$$

(It is possible to construct generalized force $f$ conjugated to the chosen response $d$ [2]. In our case $f \sim(v \cdot \varepsilon / \omega)^{I / 2}$.

Here $\varepsilon=(1 / c) \cdot(\sqrt{ } \xi \cdot \mu) \cdot E \cdot H \quad$ - electromagnetic energy density. $E \sim$ and $H \sim e+^{j \omega t}$ or $e^{-j \omega t}$ are tensions of the monochromatic field with cyclic frequency $\omega$; $v$ is volume accepting $\varepsilon$ ).

Dropping details of calculations [2] and taking into account that external perturbation is monochromatic, we will write down connection between the response $d(t)$ of the system and external perturbation $f$ in Fourier components:

$$
d_{\omega}=\alpha(\omega) \cdot f_{\omega}
$$

Here $\alpha(\omega)$ is generalized susceptibility. Symbol $\omega$ means Fourier component of value, for example:

$$
d_{u}=\int_{-\infty}^{+\infty} d(t) e^{j \omega t} d t
$$

Then, fluctuation-dissipative theorem [2] allows to write down Fourier component of square of diameter (areas) (3a) nascent hole in a kind:

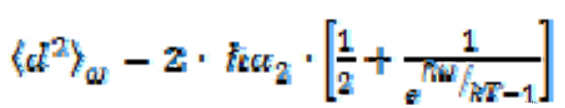

here $\alpha_{2}$ is imaginary part of generalized susceptibility (1) and (4):

$$
\alpha(\omega)=\alpha_{1}+f \alpha_{2}
$$

$\pi, k, \pi$ are constants Plank, Boltzmann and 3,14., T is an absolute temperature. The important feature $\left\langle d^{2}\right\rangle_{\omega}$ consists of that it, practically, is composition of area of the threshold hole, as functions of temperature, on a delta function $\delta(\omega)$ (appendix of B).

\section{Solution and Results}

\subsection{Determination of critical temperature}

Inverting expression (5), we will find the temperature of the electron-ion system depending on the "diameter" of the threshold hole

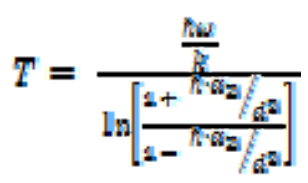

A feature of expression (6) is in what it decomposes on the "bunch" of lines - dependences of imaginary part of generalized susceptibility for different diameters versus temperature, pис.2. The curves of this bunch intersect nowhere, and each is begun with the minimum of real value.. Each of curves corresponds only to one certain

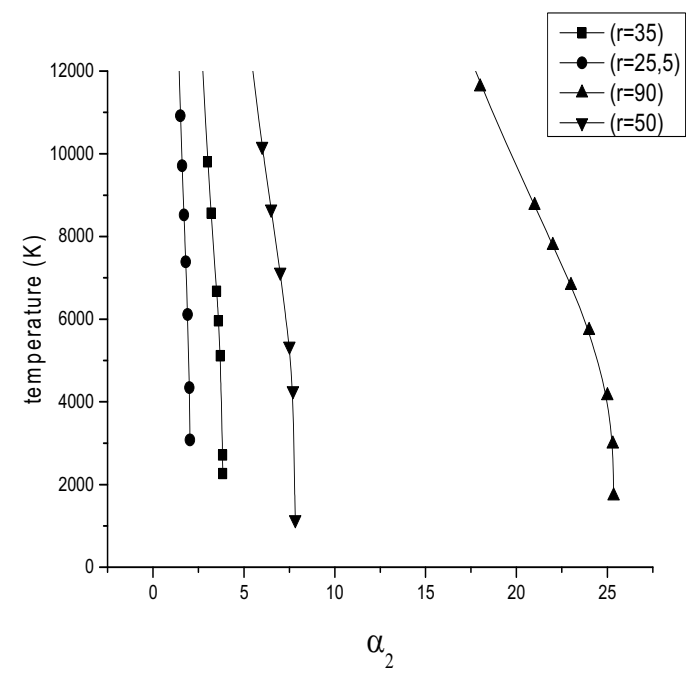

Fig.2. The temperature of the electron-ion system depending on $\alpha_{2}$ is presented as a bunch of lines for every diameter. There are lines (for four diameters) of from a complete bunch. 
diameter of exit hole. The analysis of behavior of these curves allows to find the real value of critical temperature (within the framework of the chosen approaching), and also, values of imaginary part of generalized susceptibility, $\alpha_{2}$, at different temperatures. . Research of each of lines of bunch, fig. 2, finds out on them inflection points, fig. 3. An inflection point on dependence of $d T / d \alpha_{2}$ indicate on that, how energy only of one quantum, $\hbar \omega$, is adopted by the system at temperature of $\mathrm{T}$, equaled to critical temperature of $T_{c \mathrm{r}}$.

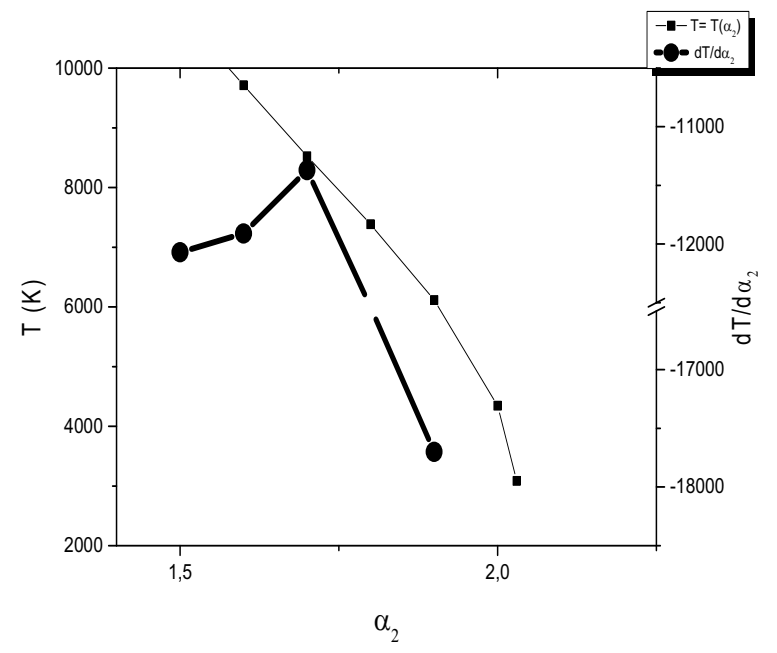

- Figure 3. Inflection point on a derivative $d T / d \alpha_{2}$. Squares designate dependence of $T$ on $\alpha_{2}$ for $\mathrm{d} \sim$ $25,5 \mu \mathrm{m}$. Mugs are the first derivative $d T / d \alpha_{2}$

Value, reciprocal to $d T / d \alpha_{2}$, i.e. $\frac{d \alpha_{\mathrm{N}}}{d T} \sim\left(\frac{\hbar \omega}{k T}\right) \cdot \frac{1}{T}$, is analogue of heat capacity at permanent pressure [ 5 ]. A characteristic peak or inflection point on dependence of $T$ versus $\alpha_{2}$ determines the value of critical temperature, рис.3. . Thus, a critical temperature for a copper is equal to $T_{\text {or }}=8522 E$

\subsubsection{State of the electron-ion system}

Imaginary part of susceptibility, $\alpha_{2}$, is fully determined by the state of electronic subsystem and its interaction with an ionic subsystem.

Behavior of the two subsystems depends on the obtained energy amount at interaction with laser field, which, in turn, depends on a reflectivity (absorptions) coefficient. Now, knowing dependence (6) and fig. 2, 3, it is possible to specify behavior of the electron-ion system at critical temperature and near it.

Within the framework of Drude approaching [6-9] it is possible to determine the reflectivity of metals $[7,8]$. The temperature range of research of reflectivities for metals is bounded enough, both in a theoretical and in experimental plan [10-12].

To calculate reflection coefficient we take into account our experimental data of response and with using (6).
Within the framework of Drude approximation an dielectric permittivity $\varepsilon(\omega)$ is expressed through external field frequency, $\omega$, plasma frequency $\omega_{p}$ and electron-ion collisions frequency of $v$ [3, 6-13]:

$$
a(\omega)=1-\frac{\omega_{p}^{2}}{\omega \cdot(\omega+\eta \omega)}=a_{1}(\omega)+f a_{2}(\omega)
$$

(within the framework of Drude model, a value $v$ depending on a context is also mentioned as mean time of relaxation of electronic distribution [11] or $v^{-1}$, and as time of relaxation of electrons at interaction both with electrons and with ions [7]).

From another side, permittivity $\varepsilon(\omega)$ and susceptibility $\alpha(\omega)$ are connected by relationships $[6,7]$ :

$$
s(\omega)=1+4 \pi \cdot \alpha(\omega)=1+4 \pi\left(\alpha_{1}+j \alpha_{2}\right)
$$

Therefore, from (6) and (7) we find that $\varepsilon_{1}$ and $\varepsilon_{2}$ :

$$
\left\{\begin{array}{c}
a_{1}=1+4 \pi \cdot \alpha_{1}(\omega) \\
a_{2}=4 \pi \cdot \alpha_{2}(\omega)
\end{array}\right.
$$

Coming back to (7) we find:

$$
\left\{\begin{array}{l}
a_{1}=1-\omega_{p}^{2} /\left(\omega^{2}+v^{2}\right) \\
a_{2}=\omega_{p}^{2} \cdot v / \sigma\left(\omega^{2}+v^{2}\right)
\end{array}\right.
$$

Here $\omega$ is frequency of the external field of radiation (radiations of laser), for a ruby laser $27 \cdot 10^{14} \mathrm{~s}^{-1}, \omega_{p}$ is plasma frequency; $\quad v$ is frequency of electron-ion interaction.

In the threshold breakdown of the through hole conditions of, [1], temperatures of experiment achieve an about a few thousand kelvin, so that $\hbar \omega \sim k T$.

At that rate $\left|\varepsilon_{1}\right| \sim\left|\varepsilon_{2}\right|$ are realized. Applications and calculations of conductivity and reflectivity coefficient connected by approaching $\left|\varepsilon_{l}\right|<<\left|\varepsilon_{2}\right|$, [13], are not carried out. Electron-ion frequency $v$ is expressed from second equation of ( $7 \mathrm{a})$ system:

$$
v^{2}-\frac{x^{2}}{a^{2}} \cdot v+\omega^{2}=0
$$

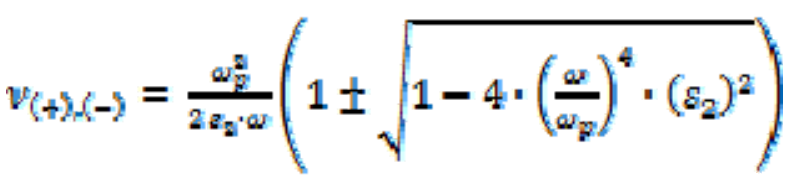

The equation shows that frequency of electron-ion interaction has two roots. These two roots testify two different states of electronic subsystem

First of roots ( + before a root) talks that the electrons of conductivity with the increase of temperature more often collide with ions (mean free time of these electrons diminishes sharply). The system is kept in metallic state. 
The second root talks that frequency of collisions falls. And it is possible (at temperatures below than critical, when the volume, occupied by the system also below, than at a critical temperature) only at reduction of number of electrons participating in conductivity. Reduction of number of electrons of conductivity talks that they (electrons) are localized on ions (or original ionic bubbles). Thus, a metal passes to the dielectric state.

. Because these both roots are equal in rights and coexist at identical temperatures, then it remains to suppose that we are at the coexistence of two states: liquid metallic and dielectric Thus there is the only condition - a critical temperature, pressure and volume, is a critical state of substance (or critical point) at which these two roots coincide:

$$
v_{(t)}=v_{(-)}
$$

Equality (11) means that subduplicate expression is equal to the zero. The condition (11) is reduced to the zero of subduplicate expression in (10a):

$$
\omega_{p}^{4}-\left(2 s_{2} \omega^{2}\right)^{2}=0
$$

or

$$
a_{2}=\frac{1}{2} \cdot\left(\frac{\omega_{p}}{\omega}\right)^{2}
$$

The value $\varepsilon_{2}$ is found from imaginary part of susceptibility $\alpha_{2}$ (9), which corresponds to critical temperature, fig.3. From (10a) subject to condition (11), taking into account (12), the electron-ion colliding frequency at critical temperature is found $v_{(+))}=v_{(-)}=v_{\text {: }}$

$$
v=\frac{\omega_{p}^{2}}{a_{\mathrm{n}} \cdot \omega}=\omega
$$

Such situation implies that external perturbation at a critical temperature distributes electrons and ions so, that frequency of their interaction becomes equal frequency of external field. Under act of the field of radiation of ruby laser $\left(\omega=27 \cdot 10^{14} \mathrm{~s}^{-1}\right)$ on a metal, at a critical temperature, this frequency equals to $v=27 \cdot 10^{14} \mathrm{~s}^{-1}$

\subsection{Temperature behavior of parameters of the electron-ion system}

\subsubsection{Reflectivity coefficient}

In order to determine reflectivity (appendix of C) and its temperature dependence it is necessary to know temperature behavior of permittivity, plasma frequency, frequency of electro-ion interaction. These values are bound by the standard set of relationships (7) - (9), which include imaginary part of susceptibility $\alpha(\omega)$. Therefore experimental dependence of temperature versus imaginary part of susceptibility, (fig.2, fig.3), allows to find temperature dependences $s_{2}, \omega_{p}$ (fig. 4), $v$ (fig. 5) and reflectivity coefficient(fig. 6) (appendix of C).

\subsubsection{Plasma frequency}

Temperature behavior of plasma frequency, $\omega_{p}$ plays an important role at consideration of reflectivity. $\omega_{p}$ (рис.4) is determined from next considerations.- It is considered that copper atoms are singly ionized (valence of $Z=1$ ) in from melting temperature up to the critical one. Then we accept $\omega_{p}=4 \pi \cdot n \cdot{ }^{2} / \mathrm{m}=31,80795 \cdot 10^{-8} \cdot \mathrm{n}$, where $\mathrm{n}=$ $6,022 \cdot 10^{23} \cdot Z \cdot \rho / A ; A=64$. Temperature dependence of copper density, $\rho$, is adopted from [16]. The measured values of metal density are taken into account up to the temperature of boiling only, inclusive. The value $\omega_{p}$ was determined from (12) at the critical temperature $\mathrm{T}_{\mathrm{cr}}=$ $8522 \mathrm{~K}$, got from our results, fig.3, preliminary defining $\varepsilon_{2}$ on $\alpha_{2}$. In addition, experiment determined imaginary part of susceptibility and, accordingly, to the permittivity, and the value of critical temperature do not allow to fluctuate of plasma frequency, due to correlation (10).

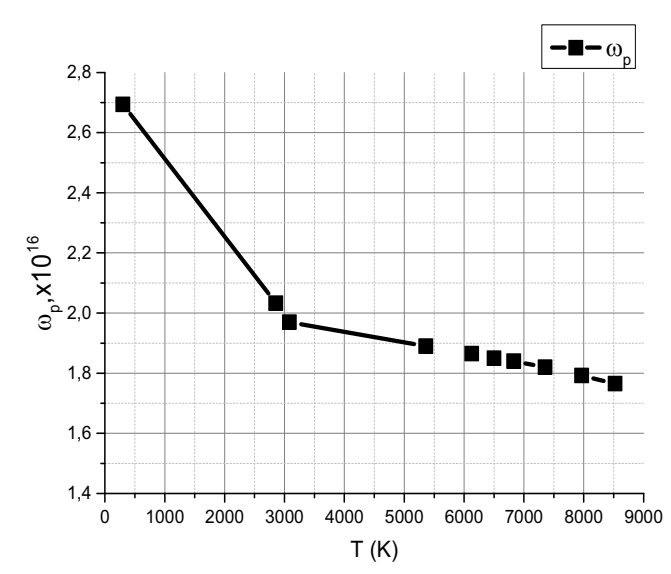

Fig.4.Temperature behavior of plasma frequency $\omega_{\mathbf{p}}$.

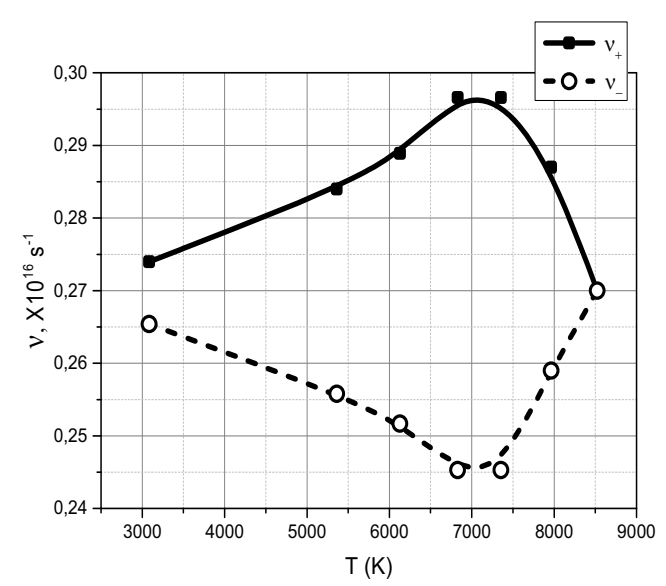

Fig. 5. Frequency $v$ is temperature dependence of electron-ion interaction .Upper curve corresponds $v(+)$ - металлической составляющей электрон-ионной системы, $v(-)$ corresponds to "dielectric" part of the electron-ion system. 


\subsubsection{Electron-ion interaction frequency}

Electron-ion interaction frequency $v$ consists of two branches $v(+)$ and $v(-)$, fig.6. First of the branches corresponds to "metallic»" part, and second - to "dielectric" part of the electron-ion system at the examined conditions. $v(+)$ illustrates the growth of frequency of electron-ion collisions, and $v(-)$ is reduction of frequency of электрон-ионных collisions due to localization of electrons by the capture of them on ionic bubbles. It leads to growth of dielectric constituent of a metal.

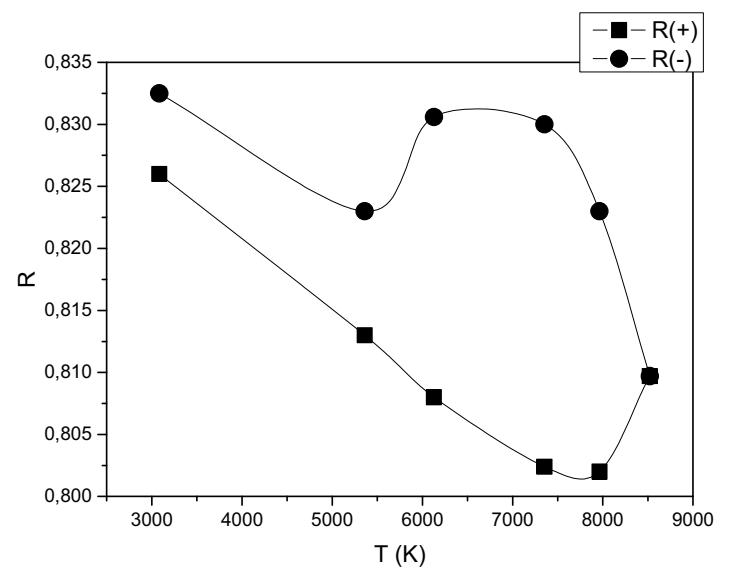

Fig.6. Reflectivity coefficients R (+) and R (-) for metallic and dielectric constituents of the electron-ion system

\subsubsection{Critical temperature}

In literature, there were a few different values of critical temperatures for a copper. A critical temperature was measured by the method of explosion of thin wire by a large current [14]: $\mathrm{T}_{\mathrm{cr}}=8390 \mathrm{~K}$. In [15] a theoretical value $\mathrm{T}_{\mathrm{cr}}=7800 \mathrm{~K}$ is got from the calculations of modelling a phase diagram. But in such calculations, if reflectivity (absorptions) were used, then for such temperatures they would be got only by extrapolation on high temperatures, as a direct measuring of these coefficients at high temperatures, higher $3000 \mathrm{~K}$ in literature were not met. In [16] it was got $\mathrm{T}_{\mathrm{cr}}=9000 \mathrm{~K}$ but by extrapolation of density of the gas state of copper from the boiling temperature

\subsubsection{Reflectivity coefficient}

$\mathrm{R}$ calculated in accordance with the appendix C. Fig.6 demonstrates complication of behavior of reflectivity in the interval of from boiling up to the critical temperature of phase transition of liquid metal to gas state. Dielectric constituent part of reflectivity $\mathrm{R}($-) plays substantial role. - In a liquid metal near-by the critical temperature of $\mathrm{R}(-)$ is substantially larger than reflection of $\mathrm{R}(+)$ caused by a metallic constituent. Quite possible, that such situation is caused not simply by appearance of the constrained localized states of electrons on ions or ionic bubbles, but also by origin of overlapping between such constrained states [22, 23] leading to generalization of the localized states. In such regions electrons work again as in a metal. Herein, presumably, and essence of the critical phenomenon at what distinction between a liquid metal and gas disappears.

\section{Conclusion}

The idea of threshold breakdown of the through holes and application of fluctuation-dissipative theorem allowed to find the value of critical temperature, and also temperature dependences of the generalized susceptibility of the system. This combination opens the prospects of more thin and detailed high temperature measuring and possibilities of management by the electron-ion system at high temperatures

\section{Appendix A}

To estimate the mean (quantum mechanical) value of $d$ from (1) and (3), we will take into account next moments: (1). At the densities of liquid metal and temperatures in thousands of Kelvin, coulomb energy of interaction of electrons and ions is comparable with their kinetic energy of motion [4]. In these conditions a liquid metal is the electron-ion system, being mixture of electron-ion gas (constrained electron-ion pairs) and electron-ion liquid. Such state is substantially heterogeneous and non-ideal. The last properties suppose a presence, both disconnected electrons and ions, providing the metallic state ("zone of conductivity" and level of Fermi in her), and appearing electron-ion pairs (atomic metallic gas) that can decompose in next moments. And at approaching of the liquid metallic state to the critical condition (to the critical temperature) frequency of origin of such virtual pairs grows (см.for example, puc.5,behaviorv(-))...... (2). In such electron-ion system, electrons can be considered, as electrons of the strongly excited state of atom at which peripheral electron of atom appear far remote from a positive ion [17] This situation is equivalent to what to electron being at long distance from a nucleus may attribute the large main quantum number $n$ (and orbital number $l=0$. So the electron corresponds to excited $S$-state). Motion of such electron (or such ion in the field of electron) can be considered as mutual motion in the coulomb field of atomic remain (ion) with the effective charge of $Z=1$ [17]: electric-field tension arising up between the charges at that rate $E_{n, l, m} \cdot(a \cdot e)^{-1},\left(E_{n, l, m}\right.$ is own energy of electron, $e$ is a electron charge, $a$ is a Bhor radius).................... (3). The conduction band in a copper appears as a result of hybridization $4 \mathrm{~s}$ and $3 \mathrm{~d}$ shells of atom [18]. So that the wave function of electron in the conductiion band can be decomposed on ball functions with certain

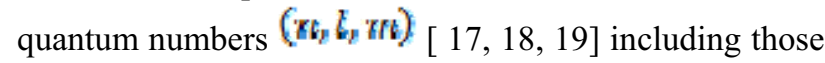
that correspond to the states of electrons participating in interaction with the external field, Part of them 
participates in dispersion of the external field, another part - in absorption (in optical transitions). For example transitions $3 d^{10} 5 s-3 d^{10} 4 p, 3 d^{10} 6 s-3 d^{10} 4 p$, $3 d^{10} 5,6,7 d-3 d^{10} 4 p$ [20], with the corresponding to them values of energy $\sim 1,0 ; 2,9 ; 3,3 \mathrm{eV}$ correspond to the optical transitions.

Taking into account these facts, (1) - (3), we can do next estimations

(a). Remoteness of electron from a nucleus in approaching of hydrogen-like atom $[17,19]$ is determined by quantum mechanical mean distance of electron in an atom

$$
\langle r\rangle_{m, l}=\frac{a}{2 \cdot g} \cdot\left[3 n^{2}-l \cdot(l+1)\right] \sim d / 2
$$

Then distance of $5 \mathrm{~S}$-state (at $\mathrm{Z}=1$, look higher, point (2)) equals to $\langle r\rangle_{\mathrm{E}, l=0}=19,838 \cdot 10^{-9} \mathrm{~cm}$, i.e., an electron is remote from a nucleus on mean distance $19,838 \cdot 10^{-9} \mathrm{~cm}$ (These distances are less at taking into account Thomas-Fermi approaching [21]) Tension of electric-field of such electron operating on rest atom (ion) equaled to $E_{n, l, m} /\left(\theta \cdot\left\langle r^{\prime}\right\rangle_{5,0}\right)$ is the same, what $\frac{1}{2} \cdot \frac{\theta_{p_{z}}}{g_{n}} \approx 3,5 \cdot 10^{7} V / \mathrm{cm}=-E$

(b). The electromagnetic field of laser radiation falling on a target is changing slower than time of electronelectron $(\tau-s)$ interaction (period of oscillation for a ruby laser $\tau=23 \cdot 10^{-16} \mathrm{~s}$ and $\omega_{p}^{-1} \sim_{p-\varepsilon}=2.32 \cdot 10^{-16} s$, where $\omega_{p}$ is plasm frequency). It implies that an electronic subsystem will have time to be tuned under external influenceю Thus electrical fields of interaction between charged particles will look like slowly changing. Atom of copper in exited state is considered in hydrogen-like atom approaching. Character (potential) of electric field allows to split up of wave function of atom $\Psi\left(x_{\alpha} y_{\alpha} z_{\alpha} i X_{\nu} Y_{\eta} Z\right)-\psi_{\nu}\left(x_{n} y_{\nu} z\right) \cdot \Phi\left(X_{\nu} Y_{z} Z\right)$ in the adiabatic approaching on the wave function of electron $\varphi_{e}\left(x_{v} y_{z} z\right)$ (here $a$-нумерует electrons in an atom; $(\mathrm{x}, \mathrm{y}, \mathrm{z})$ are coordinates of relative motion of electron, $(\mathrm{X}, \mathrm{Y}, \mathrm{Z})$ coordinates of centre-of-mass of atom ) and wave function of ion of atom $\Phi_{{ }_{n}}(X, Y, Z)$ so, that equalization of Schrodinger for the ion of atom will be written down, as

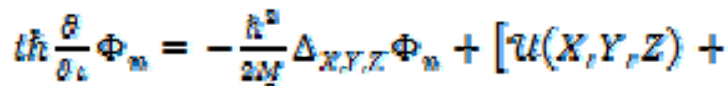

$$
\begin{aligned}
& \left.E_{n, l m}(X, Y, Z)\right] \Phi_{n}
\end{aligned}
$$

Because de Broglie wavelength of ion of atom is very small a wave function for him is searched in a kind [17 ]

$$
\Phi_{n}=A \cdot \exp \left(-t S / F_{k}\right)
$$

Where $\mathrm{S}$, is an action [17 ], equals to

$$
S=H \cdot t-P_{Z} \cdot X-P_{Y} \cdot Y-P_{Z} \cdot Z
$$

Substitution of (A2) in (A1) with an account (A3) leads to hamiltonian

$$
H=\vec{P}^{2} / 2 M+U(X, Y, Z)+E_{n, l m}(X, Y, Z)
$$

It corresponds to classic behavior of ion of atom of copper, in the external field created by surroundings of ion, $U(X, Y, Z)$ and by field, $E_{n, i, m}(X, Y, Z)$, created by remote valency electron.

We consider that $E_{\mathrm{n}, \mathrm{m}}\left(X_{v} Y_{v}, Z\right)$ at moving of centremass does not change. The external field for the ion of $U(X, Y, Z)$ is expressed through the difference of chemical potentials of electrons in the "interface" and in a "volume"

\section{$u(X, Y, Z)=\left(h_{e_{-} \text {imterfaee }}-h_{e_{-} \text {nohume }}\right)=e$ ( $\left.\varphi_{\text {interface }}-\varphi_{\text {nolume }}\right)$}

For simplicity it is accepted the vector of tension of electric-field is directed on a normal to the "interface", fig.1. Then equations of motion of centre-of-mass of remain of copper atom take the following form:

$$
\begin{gathered}
M \frac{d^{2} Z}{d t^{2}}=0 \\
M \frac{d^{2} Z}{d t^{2}}=0 \\
M \frac{d^{2} Z}{d t^{2}}=-\frac{g}{g Z}\left(U(X, Y, Z)+E_{m, b, m}(X, Y, Z)+\frac{q^{2}}{4 m \sigma \omega^{2}} J\right) \\
\text { Value }|V| \ll|U(X, Y, Z)|, \text { where } V=\frac{q^{2}}{40 m \alpha^{2}} E^{2} .
\end{gathered}
$$


It is accepted the initial value of speed of remain in the moment of $t=0$ is equal to the zero $\left(\frac{\Delta I}{d t}, \frac{d Y}{d t}, \frac{d Z}{d t}\right)=0$.

. From it is followed, the conserved values are $X-X_{0} Y-Y_{0}^{*} \sim\langle\gamma\rangle_{\mathrm{N}, l} \quad$ and $\left(X-X_{0}\right) \cdot\left(Y-Y_{0}\right)$. In other words, an area $\left(X-X_{0}\right) \cdot\left(Y-Y_{0}\right)=\sigma$, is equal to the area formed by the projection of atom on normal to moving of atom of plane, is conserved. Values of $M \cdot \sigma$ and $N \cdot M \cdot \sigma$ also are the invariants of motion during the process of transition of remain of atom from one position in other. Distance overcame by the remain of atom is

$$
\begin{aligned}
& Z-z_{0}--\frac{1}{2 M}\left[\frac{\theta}{g z}(u(X, Y, Z)+\right. \\
& \left.\left.E_{n, l m}\left(X_{t} Y_{t} Z\right)\right)\right] \cdot \tau^{2}
\end{aligned}
$$

If to accept, that

$$
\begin{aligned}
& Z-Z_{0}=\langle r\rangle_{4, i=1}-\langle r\rangle_{8, i=0}=\mid 7,671 . \\
& \left.10^{-9}\right|_{\mathrm{cm}}
\end{aligned}
$$

then the remain of atom will overcome this distance after $\sim 10^{-14} s$, before will go across from the state $\mid n=5, l=0)$ in $(n=4, l=1 \mid$

\section{Appendix B}

Calculation of spectral density of square of diameter of the threshold breakdown of the through hole,$\left.d^{2}\right\rangle_{\mathrm{a}}$

Formation of hole is the temporal phenomenon. And the diameter of minimum exit hole depends on a diameter in the moment of time previous to the breakdown. So that it is possible to talk about temporal' correlation function

$$
\left\langle d(t) \cdot d\left(t^{2}\right)\right\rangle \quad(\mathrm{B} 1)
$$

. in which $t^{\prime}$ is time moment of through breakdown of minimum exit hole by the diameter of $d . t=0$ is the preceding moment of time is chosen as point of reference. It means an exit hole arises up at $t^{\prime}-0=\tau$. Spectral density of square of diameter of the through breakdown of minimum exit hole with respect to [2] is written down:

$$
\left\langle d^{2}\right\rangle_{\alpha}=\int_{-\infty}^{+\infty}(d t(0) \cdot d(\tau)\rangle_{e^{f \omega t}} d t
$$

It is assumed that diameter during $\tau$ is changed poorly the $d(\tau)$ is decomposed into row:

$$
d(\tau)=d(0)+d \cdot \tau
$$

(B2) is rewritten with taking into account (B3)..

$$
\begin{aligned}
& \left\langle d^{2}\right\rangle_{\omega}=\int_{-\infty}^{+\infty}(d(0) \cdot(d(0)+d \cdot \tau)\rangle^{j \omega t} d t= \\
& =\int_{-\infty}^{+\infty}(d(0) \cdot d(0)\rangle e^{j \omega t} d t+\int_{-\infty}^{+\infty}(d(0) \cdot d \cdot \\
& \tau)\rangle e^{j \omega t} d t=
\end{aligned}
$$$$
\begin{aligned}
& =\langle d(0) \cdot d(0)\rangle \int_{-\infty}^{+\infty} e^{k t} d t+\tau \cdot \int_{-\infty}^{+\infty}(d(0) \\
& d(0)) e^{t \omega t} d t=
\end{aligned}
$$

the one should notice

$$
\begin{gathered}
\int_{-\infty}^{+\infty} d n d t-\Phi(\omega) \\
\int_{-\infty}^{+\infty}(d(0) \cdot d(0)) e^{j u t} d t=\langle d(0) \cdot d(0)\rangle
\end{gathered}
$$

Continuing equality (B4) and taking into account relations (B5) and (B6), then (B4) :

$$
=\langle d(0) \cdot d(0)\rangle \cdot \partial(\omega)+\tau \cdot\langle d(0) \cdot d(0)\rangle
$$

It is assumed that change of diameter $d$ versus time and size of diameter $d$ in one and the same moment of time are not correlated. Then the second member in (B7) equals to the zero. So finally:

$$
\left\langle d^{2}\right\rangle_{\omega}=d^{2}(0) \cdot \delta(\omega)
$$

\section{Appendix C}

Reflectivity coefficient (relation of Frenel of electromagnetic radiation for the normally falling on a interface ) 


$$
\begin{aligned}
& R=\left|\frac{h^{2}-1}{\sqrt{2}+1}\right|^{2}=\left|\frac{n-k \cdot k-1}{n+k \cdot k+1}\right|^{2}=\frac{(n-1)^{2}+k^{2}}{(n+1)^{2}+k^{2}} \\
& g^{2}=n-j \cdot k \\
& n=\left(\frac{1}{2} \cdot\left[a_{1} \mid\left(a_{1}^{2} \mid a_{2}^{2}\right)^{\frac{2}{2}}\right]\right)^{\frac{1}{2}} \\
& k=\left\{\frac{1}{2} \cdot\left(a_{1}^{2}+a_{2}^{2}\right)^{\frac{2}{2}}-a_{1}\right\}^{\frac{6}{2}} \\
& a=a_{1}+f \cdot a_{2}=1-\frac{\omega_{p}^{2}}{\omega^{2}+v^{2}}+f \cdot \frac{\omega_{p}^{2} v}{\left(\omega^{2}+v^{2} \eta \cdot \omega\right.}= \\
& =1-\frac{\omega_{p}^{2} \cdot \omega-j \omega_{p}^{2} \cdot v}{\left(\omega^{2}+v^{2}\right) \cdot \omega}=1-\frac{\omega_{p}^{2} \cdot(\omega-j v)}{(\omega+j v) \cdot(\omega-j v) \cdot \omega} \\
& =1-\frac{\omega}{\omega \cdot(\alpha+\mu v)}
\end{aligned}
$$$$
a_{2}=4 \pi \cdot a_{2}
$$

\section{References}

1. E.V Kalashnikov, M. Yu Kantor, A.A Bugayev,D.V Kouprienko, A.V. Chikiryaka. Part 1

2. L.D. Landau and E. M Lifshitz, Statistical Physics, Elsevier .

3. D Bauer. Theory of intense laser-matter interaction. Max-Plank-Institute für Kernphysik, Heidelberg, Germany. 2006, 106 p.

4. Ya.B. Zel'dovich., Yu.P. Raizer. Physics of Shock Waves and High-Temperature Hydrodynamic Phenomena. DOVER PUBLICATIONS, INC. Mineola, NY, 2002

5. H.E Stanley. Introduction to phase transitions and critical phenomena. Clarendon Press. Oxford 1971

6. M.S. Dresselhaus. Optical Properties of Solids. Solid State Physics. Part II, 194 (1985).

7. D Arovas. Lecture Notes on Condensed Matter Physics. Departm.of Physics. University of California, San Diego. 2010, 179 p.

8. O.V Vaculenco., V.S Severin. Semiconductor Physics, Quantum Electronics and Optoelectronics, 10, 55, (2007).

9. K Fleischer., S Chandola., Th Hermann., N. Esser. , W. Richter. , J.F. McGilp. Phys. Rev. B. 195432, (2006).

10. William $\mathrm{T}$ Walter. Change in reflectivity of Metals under intense Laser Radiation. Polytechnic Institute of New York. 1-35 (1981.).

11. Kikuo Ujihara. Journ. Appl. Phys.43, 2376 (1972).

12. M. Schmid, S. Zehnder., P. Schwaller., B. Neuenschwander.,M.Held., U. Hunziker.,
I.Zürcher Advanced Laser Technologies (ALT 12), 2-6 September, 2012, Thun, Switzerland. 8 pages.

13. G.G Gladush., I. Smurov. Physics of laser materials Processing. (Theory and epxperiment).SpringerVerlag Berlin Heidelberg 2011

14. V.E Fortov., A.N. Driemin., A.B. Leont'ev. Teplophys. High Temper. 13, 1072 (1975).

15. D Autique., G. Clair, D. L'Hermite, V Alexiades., A. Bogaerts. , B. Rethfeld. Journ. Appl.Physics. 114, 023301 (2013).

16. J.A Cahill., A.D. Kirshenbaum. Journ. Phys. Chemistry.66,1080 (1962).

17. L. D. Landau. and E. M Lifshitz, Quantum Mechanics, Elsevier

18. J. Friedel, : in The Physics of Metals, ed. J. Ziman, Cambridge Univ. (1969)

19. Hans A. Bethe. Quantum Mechanics of One- and Two-Electron Atoms Cornell University,

20. C.L. Lewis., G.P. Jackson., K.D. Stephen, V. Majidi., F.L King. Spectrochimica Acta. Part B.56, 481 (2001).

21. L Spruch. Rev. Mod. Phys.63, 151 (1991).

22. A.A Likal'ter. Teplophys..High Temper. 25, 424 (1987).

23. A.A. Likal'ter. Uspehy-Phys. 170, 831 (2000). 